Research Paper

\title{
The Prodomain-Containing BMP9 Produced from a Stable Line Effectively Regulates the Differentiation of Mesenchymal Stem Cells
}

Ruifang Li1,2, Zhengjian Yan²,3, Jixing Ye ${ }^{2,4}$, He Huang 5 , Zhongliang Wang2,3, Qiang Wei2,3, Jing Wang 2,3, Lianggong Zhao 2,6, Shun Lu', , Xin Wang 2,8, Shengli Tang1,9, Jiaming Fan 2,3, Fugui Zhang 2,3, Yulong Zou 2,3, Dongzhe Song2,8, Junyi Liao ${ }^{2,3}$, Minpeng Lu',3, Feng Liu'2,3, Lewis L. Shi², Aravind Athiviraham², Michael J. Lee $^{2}$, Tong-Chuan $\mathrm{He}^{2,}$ and Zhonglin Zhang ${ }^{2,9,}$,

1. Department of Neurology, Hubei Zhongshan Hospital, Wuhan, China

2. Molecular Oncology Laboratory, Department of Orthopaedic Surgery and Rehabilitation Medicine, The University of Chicago Medical Center, Chicago, IL, USA

3. Ministry of Education Key Laboratory of Diagnostic Medicine, and the Affiliated Hospitals of Chongqing Medical University, Chongqing, China

4. Department of Biomedical Engineering, School of Bioengineering, Chongqing University, Chongqing, China

5. Ben May Department for Cancer Research, The University of Chicago Medical Center, Chicago, IL 60637, USA

6. Department of Orthopaedic Surgery, the Second Affiliated Hospital of Lanzhou University, Lanzhou, China

7. Department of Orthopaedic Surgery, Shandong Provincial Hospital and Shandong University School of Medicine, Jinan, China

8. Department of Surgery, West China Hospital of Sichuan University, Chengdu, China;

9. Department of General Surgery, the Research Center of Digestive Diseases, Zhongnan Hospital of Wuhan University, Wuhan, China.

*Corresponding authors

$\triangle$ Corresponding authors: T.-C. He, MD, PhD, Molecular Oncology Laboratory, The University of Chicago Medical Center, 5841 South Maryland Avenue, MC 3079, Chicago, IL 60637, USA. Tel. (773) 702-7169; Fax (773) 834-4598; E-mail: tche@uchicago.edu. Zhonglin Zhang, MD, PhD, Department of General Surgery, Research Center of Digestive Diseases, Zhongnan Hospital of Wuhan University, 169 Donghu Road, Wuhan 430071, China. Tel/Fax: +011-86-27-67812588; E-mail: zhonglinzhang@boneandcancer.org

(C) Ivyspring International Publisher. Reproduction is permitted for personal, noncommercial use, provided that the article is in whole, unmodified, and properly cited. See http://ivyspring.com/terms for terms and conditions.

Received: 2015.07.24; Accepted: 2015.11.09; Published: 2016.01.01

\begin{abstract}
Background: BMPs play important roles in regulating stem cell proliferation and differentiation. Using adenovirus-mediated expression of the 14 types of BMPs we demonstrated that BMP9 is one of the most potent BMPs in inducing osteogenic differentiation of mesenchymal stem cells (MSCs), which was undetected in the early studies using recombinant BMP9 proteins. Endogenous BMPs are expressed as a precursor protein that contains an $\mathrm{N}$-terminal signal peptide, a prodomain and a C-terminal mature peptide. Most commercially available recombinant BMP9 proteins are purified from the cells expressing the mature peptide. It is unclear how effectively these recombinant BMP9 proteins functionally recapitulate endogenous BMP9.

Methods: A stable cell line expressing the full coding region of mouse BMP9 was established in HEK-293 cells by using the piggyBac transposon system. The biological activities and stability of the conditioned medium generated from the stable line were analyzed.

Results: The stable HEK-293 line expresses a high level of mouse BMP9. BMP9 conditioned medium (BMP9-cm) was shown to effectively induce osteogenic differentiation of MSCs, to activate BMP-R specific Smad signaling, and to up-regulate downstream target genes in MSCs. The biological activity of BMP9-cm is at least comparable with that induced by AdBMP9 in vitro. Furthermore, BMP9-cm exhibits an excellent stability profile as its biological activity is not affected by long-term storage at $-80^{\circ} \mathrm{C}$, repeated thawing cycles, and extended storage at $4^{\circ} \mathrm{C}$.

Conclusions: We have established a producer line that stably expresses a high level of active BMP9 protein. Such producer line should be a valuable resource for generating biologically active BMP9 protein for studying BMP9 signaling mechanism and functions.
\end{abstract}

Key words: BMP9; Mesenchymal stem cells; stem cells; osteogenic differentiation; recombinant proteins; conditioned medium 


\section{Introduction}

As members of the TGF $\beta$ superfamily, bone morphogenetic proteins (BMPs) play an important role in stem cell proliferation and differentiation during development [1-6]. Deletions of BMPs resulted in various skeletal and extraskeletal developmental abnormalities[3, 7, 8]. Several BMPs have been shown to regulate osteoblast differentiation of mesenchymal stem cells (MSCs) [4-7, 9]. As multipotent progenitors, MSCs can undergo self-renewal and differentiate into multi-lineages, including osteogenic, chondrogenic, and adipogenic lineages [6, 9-14].

We conducted a comprehensive analysis of the osteogenic activity of 14 human BMPs, and found that BMP9 (aka, growth and differentiation factor 2, or Gdf2) is one of the most potent BMPs in promoting osteogenic differentiation of MSCs [6, 13, 15-21]. We further demonstrated that BMP9 regulates a distinct set of downstream targets in MSCs [17-20]. BMP9 was originally identified from fetal mouse liver cDNA libraries, and is a relatively less well characterized member of the BMP family [22] even though BMP9 is highly expressed in the developing mouse liver [22, $23]$. It has been reported that BMP9 plays role in regulating glucose and iron homeostasis in liver [24, 25], acts as a potent synergistic factor for hematopoietic progenitor generation and colony formation [26], and plays a role in the induction and maintenance of the neuronal cholinergic phenotype in the central nervous system [27]. However, conflicting results have implicated BMP9 as either an angiogenesis inducer in endothelial cells [28-34] or as a potent anti-angiogenic factor [35].

Interestingly, in early studies the recombinant human BMP9 protein was shown to exert negligible osteoinductive activity in vivo [22], while we and others have demonstrated that exogenously expressed BMP9 is highly capable of inducing osteogenic differentiation $[6,9,15,16,36]$. BMPs are synthesized as precursor proteins, containing the $\mathrm{N}$-terminal signal peptide, a prodomain and the C-terminal mature peptide $[3,12,13,37,38]$. Several BMP-based products, mostly recombinant human BMP2 (rhBMP2), rhBMP7 (or osteogenic protein-1, OP-1) and bovine bone-derived BMP extracts, have been evaluated pre-clinically and clinically for applications in which bone induction is desired [6, 13, 37, 39, 40]. Both rhBMP2 and rhBMP7 are produced by using mammalian cell lines, such as Chinese hamster ovary $(\mathrm{CHO})$ cells. However, the traditional recombinant protein purification approaches failed to demonstrate the strong osteogenic activity of BMP9 [41, 42]. Although there are several commercial sources of recombinant BMP9, most of them are only produced the processed mature peptide. It remains unclear how effectively these recombinant BMP9 proteins can functionally recapitulate the endogenously produced BMP9 because BMP9 is one of the least studied BMPs and many aspects of its biological functions are yet to be fully understood $[3,12,13]$.

In this study, we sought to establish and characterize a producer cell line that stably expresses a high level of active BMP9 protein. Using the piggyBac transposon system to express the full coding region of mouse BMP9 gene, we established a stable HEK-293 line that expresses a high level of mouse BMP9. The $\mathrm{BMP} 9$ conditioned medium (BMP9-cm) was shown to effectively induce osteogenic differentiation, to activate BMP-R specific Smad signaling, and to up-regulate downstream target genes in MSCs. The biological activity of BMP9-cm was shown to be at least comparable with that induced by the AdBMP9 adenoviral vector in vitro. Furthermore, the BMP9-cm was shown to exhibit an excellent stability profile as its biological activity was not significantly affected by long-term storage at $-80^{\circ} \mathrm{C}$, repeated thawing cycles, and extended storage at $4^{\circ} \mathrm{C}$. Therefore, the reported BMP9 producer line should be a valuable resource for generating biologically active BMP9 protein to study the basic mechanism and function of BMP9 signaling in economical and convenient fashion.

\section{Materials and methods}

\section{Cell culture and chemicals}

Human HEK-293 cells were obtained from ATCC (Manassas, VA). Mouse mesenchymal progenitor cells iMEFs were established and previously characterized [43]. Both lines were maintained in complete Dulbecco's Modified Eagle's Medium (DMEM) supplemented with 10\% fetal bovine serum (FBS, Hyclone, Logan, UT), 100 units $/ \mathrm{ml}$ penicillin, and $100 \mu \mathrm{g} / \mathrm{ml}$ streptomycin at $37^{\circ} \mathrm{C}$ in $5 \% \mathrm{CO}_{2}$. The recently engineered 293pTP line was used for adenovirus amplification [44]. Unless indicated otherwise, all chemicals were purchased from either Sigma-Aldrich (St. Louis, MO) or Thermo Fisher Scientific (Pittsburgh, PA).

\section{Construction and generation of the stable HEK-293 cell line expressing high level of mouse BMP9 using the piggybac transposon vector system}

The coding region of mouse BMP9 was PCR amplified from a mouse EST clone and subcloned into our homemade piggyBac vector PBC2 [44-47]. The PCR amplified fragment and cloning junctions were veri- 
fied by DNA sequencing. The resultant plasmid was designated as PBC2-mBMP9. Detailed information about vector construction and sequences is available upon request.

To establish a stable cell line expressing mouse BMP9, subconfluent HEK-293 cells were co-transfected with PBC2-mBMP9 and pCMV-PBase (an expression vector for piggyBac transposase, PBase) using Lipofectamine ${ }^{\circledR}$ Transfection Reagent (Life Technologies, Grand Island, NY) according to manufacturer's instructions [44, 46, 47]. At 36h after transfection, the cells were subjected to blasticidin $S$ selection (at final concentration of $5 \mu \mathrm{g} / \mathrm{ml}$ ). The established stable line was designated as 293-BMP9 line. A control stable line was also established by co-transfecting PBC2 and pCMV-PBase into HEK-293 cells, resulting in the 293-Control line.

\section{Large-scale preparation of BMP9 conditioned medium (BMP9-cm)}

For large scale preparation of BMP9- $\mathrm{cm}$, the exponentially proliferating 293-BMP9 cells were freshly seeded into $150 \mathrm{~mm}$ cell culture dishes in complete DMEM at $70-80 \%$ confluence. After cells were attached (usually 2-4 hours after plating), the complete DMEM was carefully removed and replaced with $20 \mathrm{ml}$ per dish of Opti-MEM $®$ I (Life Technologies) for additional 48 hours. The culture medium, designated as BMP9-cm, was collected, centrifuged to remove cell debris, and aliquoted and stored at $-80^{\circ} \mathrm{C}$. The control medium (i.e., Con-cm) was prepared from 293-Control cells in a similar fashion.

\section{Generation and amplification of recombinant adenoviruses AdBMP9 and AdGFP}

Recombinant adenoviruses were generated using the AdEasy technology $[48,49]$. The coding region of human BMP9 was PCR amplified and cloned into an adenoviral shuttle vector for generating recombinant adenoviruses in HEK-293 or 293pTP cells [44]. The resulting adenovirus was designated as AdBMP9, which co-expresses GFP $[50,51]$. The adenovirus expressing only GFP (AdGFP) was used as controls [52, 53]. For all adenoviral infections, polybrene $(4-8 \mu \mathrm{g} / \mathrm{ml})$ was added to enhance infection efficiency as previously reported [54].

\section{RNA isolation and quantitative real-time PCR (qPCR) analysis}

Total RNA was isolated by using TRIZOL Reagents (Life Technologies) and subjected to reverse transcription with hexamer and M-MuLV reverse transcriptase (New England Biolabs, Ipswich, MA). The cDNA products were used as PCR templates. The qPCR primers (Table S1) were designed with Primer3 program for the genes of interest (approximately 150-250bp) [55, 56]. SYBR Green-based qPCR analysis was carried out by using CFX-96 Connect (Bio-Rad, CA) as described[57-61]. All qPCR reactions were done in triplicate. Mouse Gapdh was used as a reference gene.

\section{Alkaline phosphatase (ALP) assays}

The ALP activity was qualitatively and quantitatively assessed as described [62-64]. Experimentally, subconfluent iMEFs were either stimulated with conditioned medium or infected with adenoviral vectors. At the indicated time points, ALP activity was measured quantitatively using the modified Great Escape SEAP Chemiluminescence assay kit (BD Clontech) and qualitatively with histochemical staining assay (using a mixture of $0.1 \mathrm{mg} / \mathrm{mL}$ napthol AS-MX phosphate and $0.6 \mathrm{mg} / \mathrm{mL}$ Fast Blue BB salt) as described [53, 65]. Each assay condition was done in triplicate and repeated in three independent experiments. ALP activity was normalized by total cellular protein concentrations among the samples.

\section{Alizarin Red S staining for in vitro matrix mineralization}

Subconfluent iMEFs seeded in 24-well culture plates were treated with either conditioned medium or adenoviruses. The treated cells were cultured in complete DMEM containing ascorbic acid $(50 \mu \mathrm{g} / \mathrm{mL})$ and $\beta$-glycerophosphate $(10 \mathrm{mM})$ for 10 days $[66,67]$. The mineralized matrix nodules were visualized by using Alizarin Red S staining as described [68, 69]. Briefly, cells were fixed with $0.05 \%$ (vol/vol) glutaraldehyde at room temperature for $10 \mathrm{~min}$, washed with distilled water, and incubated with $0.4 \%$ Alizarin Red S (Sigma-Aldrich) for 5min, followed by being washed with distilled water. The staining of calcium mineral deposits was recorded under bright-field microscopy.

\section{Luciferase reporter assay}

Exponentially growing cells were seeded in 25 $\mathrm{cm} 2$ cell culture flasks and transfected with $2 \mu \mathrm{g}$ per flask of the BMPR-Smad responsive luciferase reporter p12×SBE-Luc using Lipofectamine ${ }^{\circledR}$ Transfection Reagent. At $16 \mathrm{~h}$ after transfection, cells were replated to 24-well plates, and then treated with conditioned medium or infected with adenoviruses. At 6, $12,24,48 \mathrm{~h}$ post treatment/infection, cells were lysed and subjected to luciferase assay using the Luciferase Assay kit (Promega, Madison, WI) [70, 71]. Each assay conditions were done in triplicate.

\section{Immunofluorescence staining}

Immunofluorescence assay was carried out as described [21, 72, 73]. Briefly, subconfluent cells were 
stimulated with BMP9-cm or Con-cm, for the indicated time the cells were fixed with methanol, permeabilized with $1 \%$ NP-40, and blocked with $10 \%$ donkey serum, followed by incubating with p-Smad1/5/8 antibody (Santa Cruz Biotechnology). After being washed, cells were incubated with a Texas Red-labeled secondary antibody (Santa Cruz Biotechnology). Stains were examined under a fluorescence microscope. Stains without primary antibodies, or with control IgG, were used as negative controls.

\section{Statistical analysis}

All quantitative experiments were carried out in triplicate and/or repeated three times. Data were expressed as mean \pm SD. Statistical analysis was done by one-way analysis of variance and the student's $t$ test. A value of $p<0.05$ was considered statistically significant.

\section{Results and discussion}

\section{High level of expression of BMP9 can be ac- complished in a stable 293 cell line engineered with the piggyBac transposon system}

Using the recombinant adenoviral vectors expressing the 14 types of BMPs in mesenchymal stem cells (MSCs), we were the first to demonstrate that BMP9 is one of the most potent BMPs to induce osteogenic differentiation in MSCs [6, 13, 15-21]. Previous attempts that failed to detect the osteogenic activity of BMP9's were largely due to the use of recombinant BMP9 proteins (especially the ones generated from

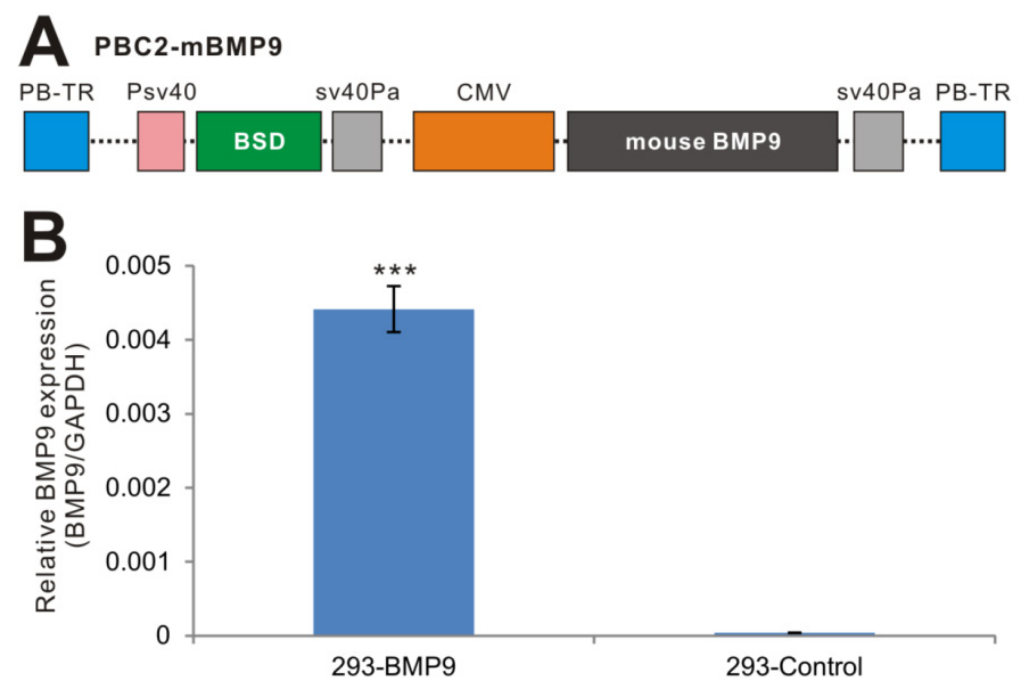

Figure 1. Establishment of stable BMP9-expressing 293 cells using the piggyBac transposon system. (A) Schematic representation of the piggyBac vector PBC2-mBMP9 that expresses mouse BMP9, along with the antibiotic resistance marker for blasticidin S (BSD). The BMP9 expression is driven the strong CMV promoter. PB-TR, piggyBac terminal repeats. (B) High level of mouse BMP9 in stable 293-BMP9 cells. Total RNA was isolated from subconfluent 293-BMP9 and 293-Control cells, and subjected to quantitative RT-PCR reactions using primers specific for mouse BMP9 coding region and human GAPDH. Relative BMP9 expression levels were calculated by dividing the relative mouse BMP9 levels with respective human GAPDH levels. Each assay condition was done in triplicate. “***”, $p<0.00 I$. prokaryotic cells) that exhibited significantly diminished biological activities, including osteogenic activity. In this study, we attempt to generate a stale mammalian cell line that produces high level of BMP9 protein in the culture medium, which can then be used for in vitro basic mechanistic studies.

While retroviral or lentiviral system is commonly-used vectors for establishing stable cell lines, we have demonstrated that the piggyBac transposon system is superior to retroviral and/or lentiviral vectors in terms of transgene expression [44, 46, 47]. Furthermore, we demonstrated that CMV is one of the strongest promoters in piggyBac-mediated transgene expression in HEK-293 cells [47]. Thus, we subcloned the full coding region of mouse BMP9 into our modified piggy $\mathrm{Bac}$ vector $\mathrm{PBC} 2$, in which $\mathrm{BMP} 9$ is driven by the CMV promoter (Fig. 1A)[44, 46, 47], resulting in PBC2-mBMP9. This vector confers blasticidin S resistance. Subsequently, the stable 293 cell line, 293-BMP9, which expresses mouse BMP9, was obtained by co-transfecting PBC2-mBMP9 and a piggy$B a c$ transposase expression vector into HEK-293 cells. The empty PBC2 vector was used to make the control cell line 293-Control.

We next tested the expression of mouse BMP9 in the stable line. Since there have not been good commercially available BMP9 antibodies for Western blotting, we examined mouse BMP9 expression using qPCR analysis, and found that the expression of mouse BMP9 in 293-BMP9 cells was 110 times higher than that in 293-Control cells $(p<0.001)$ (Fig. 1B), strongly suggesting that the 293-BMP9 stable line may produce a high level of BMP9 protein.

\section{BMP9 conditioned medium (BMP9-cm) effectively induces os- teogenic differentiation of mesen- chymal stem cells}

To determine the biological activity of the prepared BMP9-cm, we first analyzed its ability to induce early osteogenic marker alkaline phosphatase (ALP) in our previously characterized MSC line iMEFs $[43,46]$. We found that BMP9-cm induced ALP activity was readily detectable at as low as $6.25 \%$ at day 3 although there was a trend of increased ALP activity in a dose-dependent manner (Fig. 2A panel a) and time-dependent fashion (Fig. 2A panel $\mathbf{b}$ ). At the final concentration of $25 \%$, BMP9-cm induced robust ALP activity, which is comparable with that induced by AdBMP9 (at optimal MOI=10) (Fig. 2A). Quantitative analysis of the BMP9-cm induced ALP activity showed a similar 
trend as the highest ALP activities were found in the iMEFs stimulated with BMP9-cm at 50\% concentration, which is several times higher than that stimulated with AdBMP9 (Fig. 2B). These results suggest that the prepared BMP9-cm at certain concentrations may be equally effective, if more effective than, compared with the adenovirus-mediated BMP9 expression in inducing osteogenic differentiation.

We also tested the ability of the BMP9-cm to induce in vitro matrix mineralization in the MSCs. When iMEFs were simulated with BMP9- $\mathrm{cm}$, Con-cm, or infected with AdBMP9 or AdGFP, a significant amount of mineral nodules were formed in BMP9-cm stimulated MSCs, and to a lesser extent in the AdBMP9-transduced cells (Fig. 2C), suggesting that the BMP9-cm may be highly effective in inducing osteogenic differentiation of MSCs.

\section{BMP9-cm effectively activates the Smad sig- naling pathway and up-regulates the expres- sion of downstream target genes}

We further analyzed the biological activity of BMP9-cm at molecular mechanistic levels. First, we analyzed the ability of BMP9-cm to activate BMP-R specific Smad reporter, e.g., p12xSBE-Luc [74]. When the reporter-transduced iMEFs were stimulated with BMP9-cm, the luciferase activity significantly increased at as early as $6 \mathrm{~h}$ after simulation, and continued to increase up to 48 hours after stimulation (Fig. 3A, panel a). When using AdBMP9, we found that that AdBMP9 did not induce any significant luciferase activity until $24 \mathrm{~h}$ after infection although the luciferase activity was significantly higher at $48 \mathrm{~h}$ (Fig. 3B, panel b), suggesting that AdBMP9-transduced cells may continuously produce BMP9 protein and hence activate the Smad signaling pathway.

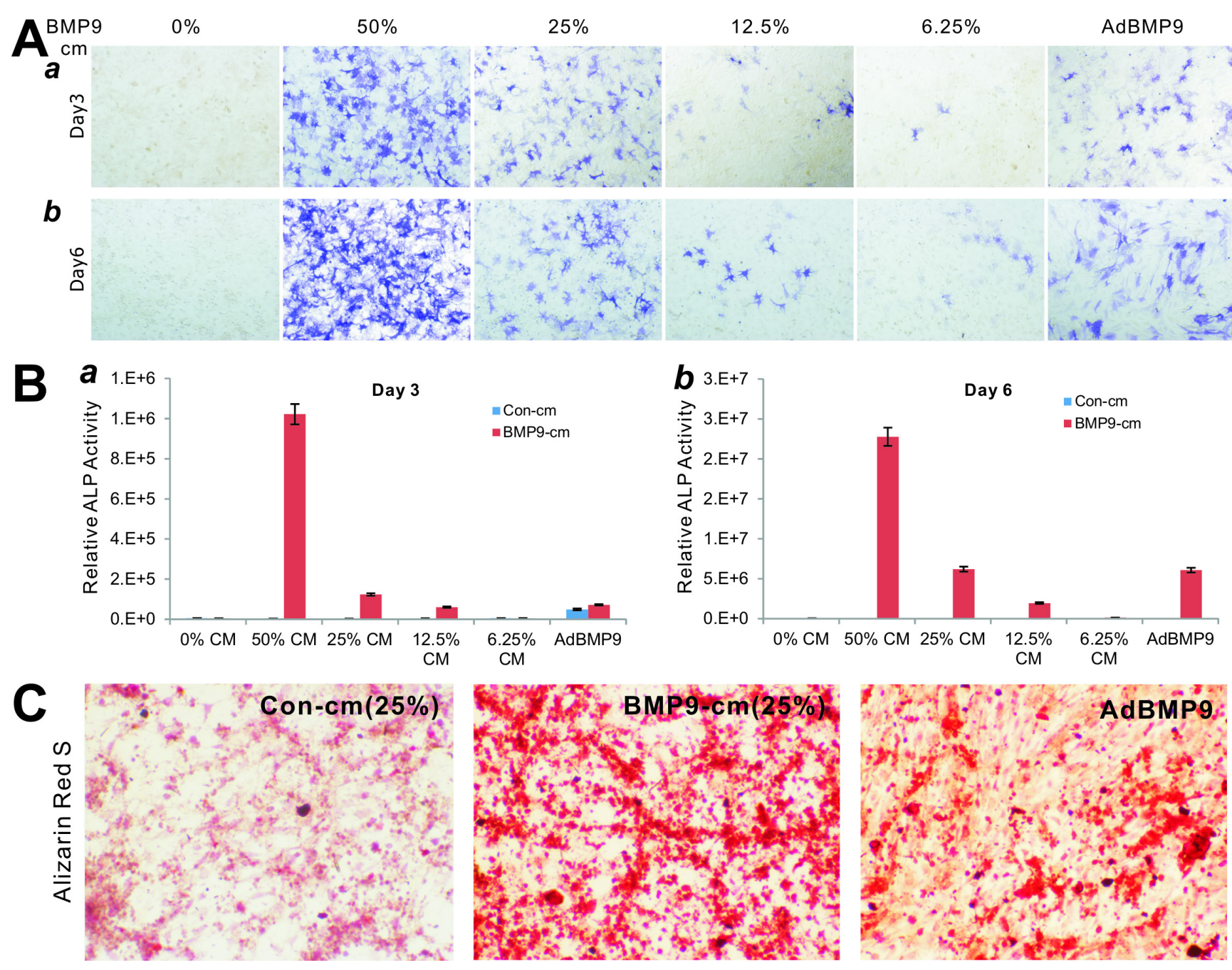

Figure 2. Induction of effective osteogenic differentiation of iMEFs by the conditioned medium prepared from 293-BMP9 cells (BMP9-cm). (A) and (B) BMP9-cm induces ALP activity in a dose-dependent fashion. Subconfluent iMEFs were seeded in 24-well cell culture plates and treated with varied concentrations of BMP9-cm or Con-cm (data not shown). At day $3(a)$ and day $6(b)$, cells were fixed for ALP histochemical staining (A) or quantitative ALP assay (B). The iMEFs transduced with AdBMP9 or AdGFP (data not shown) at $\mathrm{MOI}=10$ were used as positive and negative controls. Each assay condition was done in triplicate. Representative images are shown. (C) BMP9-cm induces effective matrix mineralization in vitro. Subconfluent iMEF cells were treated with BMP9-cm or Con-cm (at 25\%), or infected with AdBMP9 or AdGFP (data not shown), and cultured in mineralization medium for 10 days. Cells were fixed and subjected to Alizarin Red $\mathrm{S}$ staining. Each assay condition was done in triplicate. Representative images are shown. 


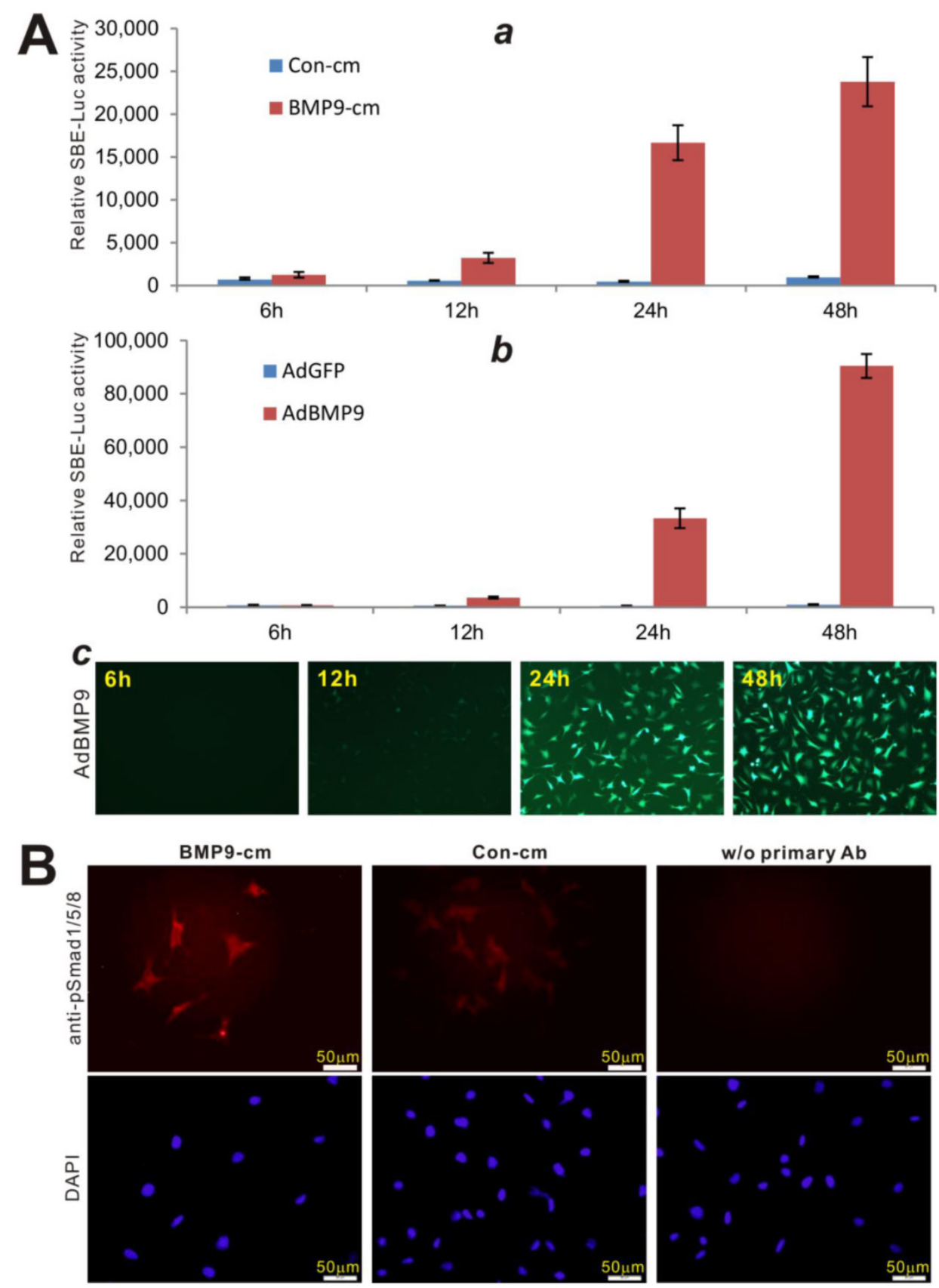

Figure 3. BMP9-cm effectively activates BMP-R specific Smad signaling pathway. (A) BMP9-cm activates BMP-R specific Smad reporter in a time-course dependent fashion. Exponentially growing iMEF cells were transfected with p12xSBE-Luc reporter plasmid using Lipofectamine Transfection Reagents (Invitrogen). The transfected cells were replated at $16 \mathrm{~h}$ post transferction, and treated BMP9-cm or Con-cm (at $25 \%$ ) (a), or infected with AdBMP9 or AdGFP (b, c) as controls. Firefly luciferase activities were assessed at the indicated time points. Each assay condition was done in triplicate. (B) BMP9-cm effectively induces phosphorylation and nuclear translocation of Smad1/5/8 in iMEFs. Subconfluent iMEFs were starved overnight and stimulated with BMP9-cm or Con- $\mathrm{cm}$ (at $25 \%$ final concentration) for $4 \mathrm{~h}$. The cells were fixed and subjected to immunofluorescence staining using an anti-pSmad1/5/8 antibody (Santa Cruz Biotechnology). Cells stained without the primary antibody was used as a negative control. Cell nuclei were stained with DAPI. Representative images are shown.

Secondly, since the phosphorylation and nuclear translocation of BMP-R specific Smad1/5/8 is considered one of the earliest BMP signaling events $[3$, $75]$, we analyzed the location of phosphorylated Smad1/5/8 protein upon BMP9-cm stimulation. Using immunofluorescence staining, we found that BMP9-cm induced an apparent increase in phosphorylated Smad1/5/8 proteins, which were mostly localized in cell nucleus (Fig. 3B), indicating that
BMP9-cm can effectively activate the BMPR-specific Smads in MSCs.

Furthermore, we determined the ability of BMP9-cm to up-regulate downstream target genes. Through gene expression profile analysis, we previously demonstrated that BMP9 can effectively induce the expression of multiple downstream target genes, including Smad6, Smad7, Id1, Id2, Id3, and CCN1 [17-19]. We stimulated iMEFs with BMP9-cm or 
Con-cm and isolated RNA samples at $12 \mathrm{~h}, 24 \mathrm{~h}$, and $48 \mathrm{~h}$ after stimulation. We also infected iMEFs with AdBMP9 or AdGFP as controls. We found five of the early target genes, e.g., Smad6, Smad7, Id1, Id2, and Id3, were significantly induced by BMP9-cm, and only CCN1 was induced at $24 \mathrm{~h}$ after BMP9-cm stimulation (Fig. 4). As expected, AdBMP9-transduced iMEFs exhibited higher levels of expression of these target genes at $48 \mathrm{~h}$ (Fig. 4). Taken together, these results have demonstrated that BMP9-cm can effectively activate the Smad signaling pathway and up-regulate the expression of downstream target genes.

\section{BMP-cm is biologically effective and stable}

We further compared the biological activity of $\mathrm{BMP}-\mathrm{cm}$ with the purified BMP9 protein and different titers of AdBMP9, in terms of inducing ALP activity. We tested three titers of AdBMP9 (Fig. 5A) and three concentrations of rhBMP9. The rhBMP9 was kindly provided by HumanZyme (Chicago, IL), which was the purified BMP9 protein from the cell medium collected from an engineered HEK-293 cell line overexpressing the full-length coding region of human BMP9. We found that ALP activities induced by BMP9-cm at $20 \%$ and $50 \%$ were comparable with that induced by rhBMP9 at $10 \mathrm{ng} / \mathrm{ml}$ and $20 \mathrm{ng} / \mathrm{ml}$, respectively (Fig. 5B), both of which were about equal or higher than that in AdBMP9-transduced cells (Fig. 5B). The slightly lower ALP activity in AdBMP9-transduced iMEFs may be caused by the fact that the ALP activity assays were conducted at an early time point (day 3 after infection) in these samples. Nonetheless, these results demonstrate that BMP9-cm is as effective as the purified BMP9 protein.
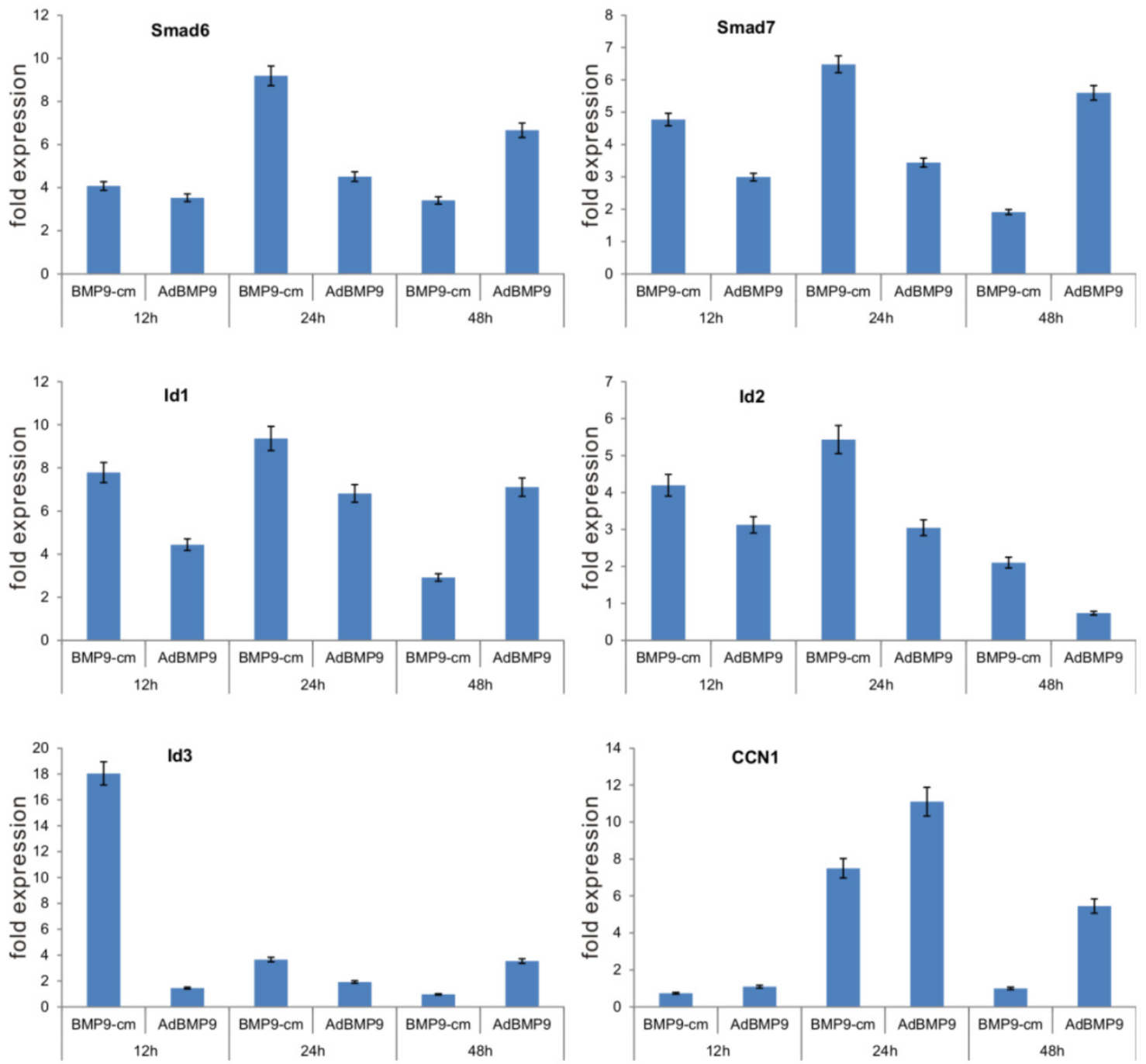

Figure 4. BMP9-cm effectively induces the expression of downstream target genes in iMEFs. Subconfluent iMEFs were stimulated with BMP9-cm or Con-cm (at $25 \%$ concentrations), or infected with AdBMP9 or AdGFP (MOI=10), and maintained in 1\% FBS DMEM. At the indicated time points, total RNA was isolated using TRIzol reagents, subjected to reverse transcription reactions, and quantitative real-time PCR (qPCR) analysis using primers specific for mouse Smad6, Smad7, Id1, Id2, Id3, and CCN1 transcripts. Fold expression was calculated by dividing BMP9-induced gene expression with the gene expression level in Con-cm or AdGFP groups. The qPCR reactions were done in triplicate. 


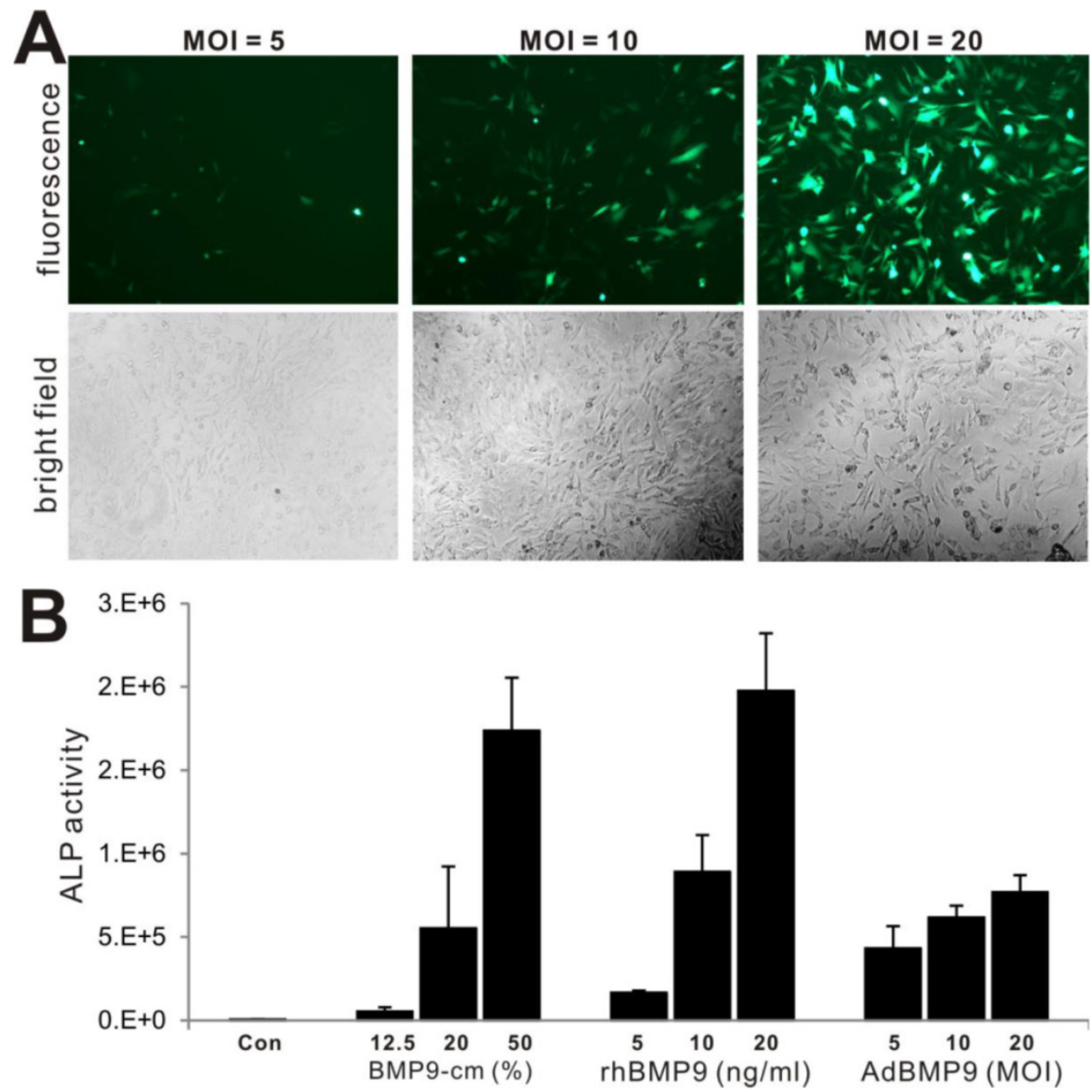

Figure 5. Comparison of BMP9-cm, rhBMP9 and AdBMP9 in inducing ALP activity in iMEF cells. (A) Subconfluent iMEF cells were infected with the indicated titers of AdBMP9 or AdGFP (data not shown). GFP signal was recorded at 24h after infection. Representative images are shown. (B) Subconfluent iMEFs were stimulated in varied concentrations of BMP9-cm or rhBMP9 or different titers of AdBMP9 or AdGFP (data not shown). At day 3, cells were lysed and subjected to ALP assays. Each assay condition was done in triplicate.

Lastly, we carried out series of experiments to test the long-term stability of BMP9-cm. We first examined the effect of long-term $-80^{\circ} \mathrm{C}$ storage on BMP9's biological activity. When the iMEFs were stimulated with the freshly prepared BMP9-cm and the BMP9-cm samples stored at $-80^{\circ} \mathrm{C}$ for 15 days and 30 days, we found that the three batches of samples induced the ALP activities at a similar level $(p=0.526)$ (Fig. 6A). We next tested the effect of repeated thawing of the BMP9-cm samples on BMP9's and found that the same preparation retained a similar level of BMP9 activity after five freezing-thawing cycles $(p=0.452)$ (Fig. 6B). Lastly, we tested the stability of the BMP9-cm preparations that were kept at $4{ }^{\circ} \mathrm{C}$ for up to one week and found that BMP9-induced ALP activity remained relatively unchanged $(p=0.612)$ (Fig. 6C). Taken together, these results demonstrate that the BMP9-cm preparations are fairly stable and suitable for long-term storage. These properties should render BMP9-cm economical and convenient for many in vitro functional and mechanistic studies of BMP9 actions.

\section{Post-translational modifications may play an important role in regulating BMP9's biological activities}

As for other members of the TGF $\beta / B M P$ superfamily, BMP9 is synthesized as a long precursor protein, containing an N-terminal signal peptide, a prodomain and a C-terminal mature peptide $[3,12,13,37$, 38]. BMP monomers are stabilized by the six-cystine knot, and BMPs are usually secreted in homomeric dimer form. The dimer is stabilized by the seventh cysteine within each monomer [76]. Serine endoproteases cleave BMP proproteins within trans-Golgi network, yielding mature protein for secretion [77]. A recent study reported that the prodomain of BMP4 is necessary and sufficient to generate stable BMP4/7 heterodimers with enhanced bioactivity in vivo [78], suggesting an important role for the prodomain in dimerization.

BMPs are intrinsically stable proteins due to their tightly folded, disulfide bond-stabilized structures $[37,39]$. Recombinant human BMP2 (rhBMP2), 
rhBMP7 (or osteogenic protein-1, OP-1), bovine bone-derived BMP extracts, were evaluated pre-clinically and clinically for several applications to enhance bone formation $[6,13,37,39$, 40]. Commonly used rhBMP2 and rhBMP7 are manufactured using mammalian production cell lines, such as $\mathrm{CHO}$ cells. As mammalian cells are used to synthesize these BMPs, the recombinant proteins should be dimerized, processed by removal of the propeptide, and glycosylated as are the naturally occurring BMP molecules.

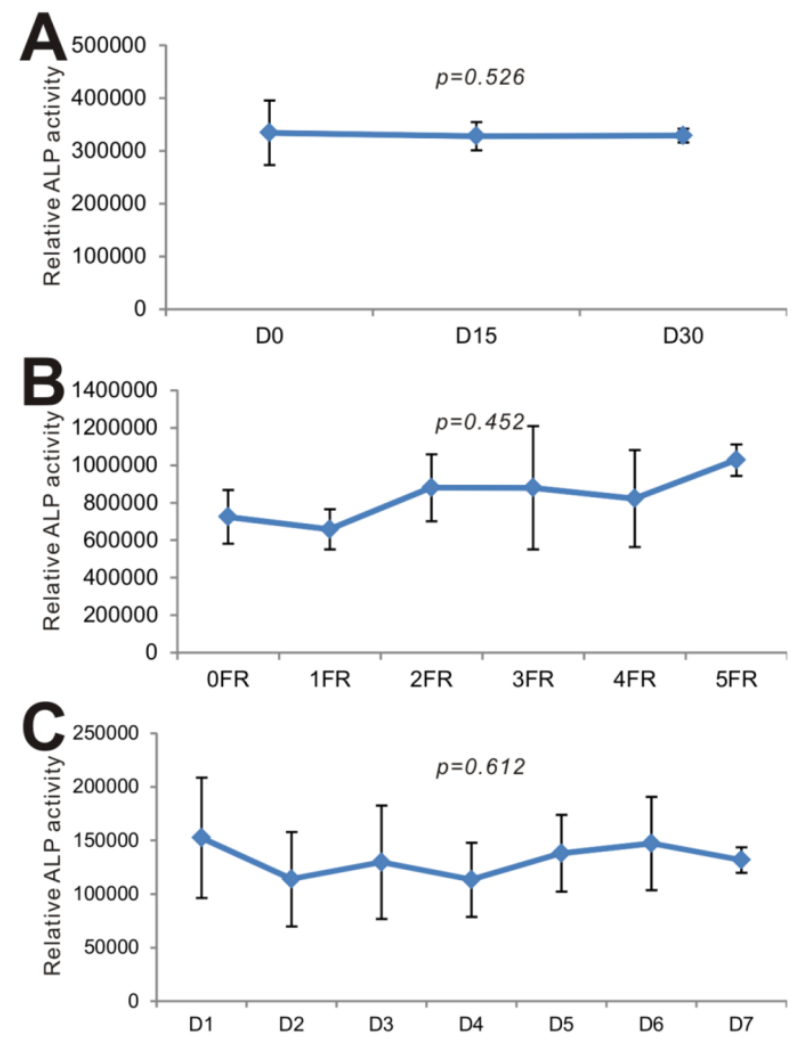

Figure 6. BMP9-cm exhibits stable long-term biological activity. (A) Effect of $-80^{\circ} \mathrm{C}$ storage on BMP9-cm's biological activity. The large-scale prepared BMP9-cm was stored in $-80^{\circ} \mathrm{C}$ freezers. Aliquots of BMP9-cm (at $25 \%$ concentration) were thawed and used to stimulate subconfluent iMEFs. ALP activity was determined after 3 days of stimulation. Assays were done in triplicate $(p=0.526)$. (B) Effect of freezing-thawing (FR) cycles on BMP9-cm's bioactivity. Aliquots of BMP9-cm were frozen at $-80^{\circ} \mathrm{C}$ and thawed at $4^{\circ} \mathrm{C}$, which constitutes one FR cycle. The aliquots were subjected to up to 5 FR cycles. BMP9-cm aliquots with varied FR cycles (at $25 \%$ concentration) were used to stimulate subconfluent iMEFs. ALP activity was determined at 3 days after stimulation. Assays were done in triplicate $(p=0.452)$. (C) Effect of $4^{\circ} \mathrm{C}$ storage time on effect on BMP9-cm's bioactivity. Aliquots of BMP9-cm were stored at $4^{\circ} \mathrm{C}$ for the indicated time points. The BMP9-cm aliquots with varied storage lengths (at $25 \%$ concentration) were used to stimulate subconfluent iMEFs. ALP activity was determined at 3 days after stimulation. Assays were done in triplicate $(p=0.612)$.

However, the traditional recombinant protein purification approaches failed to demonstrate the strong osteogenic activity of BMP9. Using adenovirus-mediated gene expression of the 14 types of BMPs in MSCs, we found that BMP9 exhibits the highest osteogenic activity both in vitro and in vivo [6, 13, 15-21]. There are several commercial sources of recombinant BMP9. However, most of these recombi- nant proteins are only produced based on the processed mature peptide sequence. Although there are some published reports using some of the recombinant BMP9 proteins, it remains unclear how effectively the recombinant BMP9 protein faithfully reproduces the endogenously produced BMP9 as BMP9 is one of the least studied BMPs and many aspects of its biological functions are yet to be fully understood $[3,12,13]$. In our unpublished studies, we found that rhBMP9 proteins from at least two different commercial sources exhibited significantly weaker osteogenic activity, compared with that induced by AdBMP9. Using the purified BMP9 produced from the full-length coding region of human BMP9 in 293 cells (provided by HumanZyme, unfortunately not commercially available yet), we found this recombinant BMP9 effectively induces osteogenic differentiation of MSCs, comparable with that induced by AdBMP9 in vitro.

\section{Conclusion}

In order to overcome the unavailability of biologically active BMP9 protein for in vitro functional and mechanistic studies, we engineered the 293-based BMP9 producer line using the piggyBac transposon system. Our results demonstrated that the prepared BMP9 conditioned medium is highly effective in inducing osteogenic differentiation of MSCs and fairly stable after long-term storage or repeated thawing cycles. Therefore, the reported BMP9 producer line should be a valuable resource for providing biologically active and stable BMP9 protein in an economical and convenient fashion.

\section{Supplementary Material}

Table S1. http:/ /www.medsci.org/v13p0008s1.pdf

\section{Acknowledgments}

The authors wish to thank Dr. Di Chen of Rush University Medical Center for kindly providing p12xSBE-Luc reporter. The authors also thank $\mathrm{Hu}-$ manZyme, Inc., Chicago, IL, for the kind provision of purified native human BMP9 protein. Work in the investigators' laboratories was supported in part by research grants from the National Institutes of Health (AT004418 to TCH), North American Spine Society $(\mathrm{TCH})$, the Chicago Biomedical Consortium Catalyst Award (TCH), and the Scoliosis Research Society (MJL). This work was also supported in part by The University of Chicago Core Facility Subsidy grant from the National Center for Advancing Translational Sciences (NCATS) of the National Institutes of Health through Grant UL1 TR000430. 


\section{Competing Interests}

The authors have declared that no competing interest exists.

\section{References}

1. Varga AC, Wrana JL. The disparate role of BMP in stem cell biology. Oncogene. 2005; 24: 5713-21.

2. Zhang J, Li L. BMP signaling and stem cell regulation. Dev Biol. 2005; 284: $1-11$.

3. Wang RN, Green J, Wang Z, Deng Y, Qiao M, Peabody M, et al. Bone Morphogenetic Protein (BMP) Signaling in Development and Human Diseases. Genes Dis. 2014; 1: 87-105.

4. Shi Y, Massague J. Mechanisms of TGF-beta signaling from cell membrane to the nucleus. Cell. 2003; 113: 685-700.

5. Attisano L, Wrana JL. Signal transduction by the TGF-beta superfamily. Science. 2002; 296: 1646-7.

6. Luu HH, Song WX, Luo X, Manning D, Luo J, Deng ZL, et al. Distinct roles of bone morphogenetic proteins in osteogenic differentiation of mesenchymal stem cells. J Orthop Res. 2007; 25: 665-77.

7. Hogan BL. Bone morphogenetic proteins: multifunctional regulators of vertebrate development. Genes Dev. 1996; 10: 1580-94.

8. Zhao GQ. Consequences of knocking out BMP signaling in the mouse. Genesis. 2003; 35: 43-56.

9. Deng ZL, Sharff KA, Tang N, Song WX, Luo J, Luo X, et al. Regulation of osteogenic differentiation during skeletal development. Front Biosci. 2008; 13: 2001-21.

10. Prockop DJ. Marrow stromal cells as stem cells for nonhematopoietic tissues. Science. 1997; 276: 71-4.

11. Pittenger MF, Mackay AM, Beck SC, Jaiswal RK, Douglas R, Mosca JD, et al. Multilineage potential of adult human mesenchymal stem cells. Science. 1999; 284: $143-7$.

12. Lamplot JD, Qin J, Nan G, Wang J, Liu X, Yin L, et al. BMP9 signaling in stem cell differentiation and osteogenesis. Am J Stem Cells. 2013; 2: 1-21.

13. Luther G, Wagner ER, Zhu G, Kang Q, Luo Q, Lamplot J, et al. BMP-9 Induced Osteogenic Differentiation of Mesenchymal Stem Cells: Molecular Mechanism and Therapeutic Potential. Curr Gene Ther. 2011; 11: 229-40.

14. Rastegar F, Shenaq D, Huang J, Zhang W, Zhang BQ, He BC, et al. Mesenchymal stem cells: Molecular characteristics and clinical applications. World J Stem Cells. 2010; 2: 67-80.

15. Cheng $\mathrm{H}$, Jiang $\mathrm{W}$, Phillips FM, Haydon RC, Peng Y, Zhou L, et al. Osteogenic activity of the fourteen types of human bone morphogenetic proteins (BMPs). J Bone Joint Surg Am. 2003; 85A: 1544-52.

16. Kang Q, Sun MH, Cheng $\mathrm{H}$, Peng $\mathrm{Y}$, Montag AG, Deyrup AT, et al. Characterization of the distinct orthotopic bone-forming activity of 14 BMPs using recombinant adenovirus-mediated gene delivery. Gene Ther. 2004; 11: 1312-20.

17. Peng $\mathrm{Y}$, Kang Q, Cheng H, Li X, Sun MH, Jiang W, et al. Transcriptional characterization of bone morphogenetic proteins (BMPs)-mediated osteogenic signaling. J Cell Biochem. 2003; 90: 1149-65.

18. Peng Y, Kang Q, Luo Q, Jiang W, Si W, Liu BA, et al. Inhibitor of DNA binding/differentiation helix-loop-helix proteins mediate bone morphogenetic protein-induced osteoblast differentiation of mesenchymal stem cells. J Biol Chem. 2004; 279: 32941-9.

19. Luo Q, Kang Q, Si W, Jiang W, Park JK, Peng Y, et al. Connective Tissue Growth Factor (CTGF) Is Regulated by Wnt and Bone Morphogenetic Proteins Signaling in Osteoblast Differentiation of Mesenchymal Stem Cells. J Biol Chem. 2004; 279: 55958-68.

20. Sharff KA, Song WX, Luo X, Tang N, Luo J, Chen J, et al. Hey1 Basic Helix-Loop-Helix Protein Plays an Important Role in Mediating BMP9-induced Osteogenic Differentiation of Mesenchymal Progenitor Cells. J Biol Chem. 2009; 284: 649-59.

21. Tang N, Song WX, Luo J, Luo X, Chen J, Sharff KA, et al. BMP9-induced osteogenic differentiation of mesenchymal progenitors requires functional canonical Wnt/beta-catenin signaling. J Cell Mol Med. 2009; 13: 2448-64.

22. Song JJ, Celeste AJ, Kong FM, Jirtle RL, Rosen V, Thies RS. Bone morphogenetic protein-9 binds to liver cells and stimulates proliferation. Endocrinology. 1995; 136: 4293-7.

23. Miller AF, Harvey SA, Thies RS, Olson MS. Bone morphogenetic protein-9. An autocrine/paracrine cytokine in the liver. J Biol Chem. 2000; 275: 17937-45.

24. Chen C, Grzegorzewski KJ, Barash S, Zhao O, Schneider H, Wang O, et al. An integrated functional genomics screening program reveals a role for BMP-9 in glucose homeostasis. Nat Biotechnol. 2003; 21: 294-301.

25. Truksa J, Peng H, Lee P, Beutler E. Bone morphogenetic proteins 2, 4, and 9 stimulate murine hepcidin 1 expression independently of $\mathrm{Hfe}$, transferrin receptor 2 (Tfr2), and IL-6. Proc Natl Acad Sci U S A. 2006; 103: 10289-93.

26. Ploemacher RE, Engels LJ, Mayer AE, Thies S, Neben S. Bone morphogenetic protein 9 is a potent synergistic factor for murine hemopoietic progenitor cell generation and colony formation in serum- free cultures. Leukemia. 1999; 13: 428-37.
27. Lopez-Coviella I, Berse B, Krauss R, Thies RS, Blusztajn JK. Induction and maintenance of the neuronal cholinergic phenotype in the central nervous system by BMP-9. Science. 2000; 289: 313-6.

28. Scharpfenecker M, van Dinther M, Liu Z, van Bezooijen RL, Zhao Q, Pukac L, et al. BMP-9 signals via ALK1 and inhibits bFGF-induced endothelial cell proliferation and VEGF-stimulated angiogenesis. J Cell Sci. 2007; 120: 964-72.

29. Cunha SI, Pardali E, Thorikay M, Anderberg C, Hawinkels L, Goumans MJ, et al. Genetic and pharmacological targeting of activin receptor-like kinase 1 impairs tumor growth and angiogenesis. J Exp Med. 2010; 207: 85-100.

30. Mitchell D, Pobre EG, Mulivor AW, Grinberg AV, Castonguay R, Monnell TE, et al. ALK1-Fc inhibits multiple mediators of angiogenesis and suppresses tumor growth. Mol Cancer Ther. 2010; 9: 379-88.

31. Suzuki Y, Ohga N, Morishita Y, Hida K, Miyazono K, Watabe T. BMP-9 induces proliferation of multiple types of endothelial cells in vitro and in vivo. J Cell Sci. 2010; 123: 1684-92.

32. Castonguay R, Werner ED, Matthews RG, Presman E, Mulivor AW, Solban N, et al. Soluble endoglin specifically binds bone morphogenetic proteins 9 and 10 via its orphan domain, inhibits blood vessel formation, and suppresses tumor growth. J Biol Chem. 2011; 286: 30034-46.

33. Park JE, Shao D, Upton PD, Desouza P, Adcock IM, Davies RJ, et al. BMP-9 induced endothelial cell tubule formation and inhibition of migration involves Smad1 driven endothelin-1 production. PLoS One. 2012; 7: e30075.

34. Yao Y, Jumabay M, Ly A, Radparvar M, Wang AH, Abdmaulen R, et al. Crossveinless 2 regulates bone morphogenetic protein 9 in human and mouse vascular endothelium. Blood. 2012.

35. David L, Mallet C, Keramidas M, Lamande N, Gasc JM, Dupuis-Girod S, et al. Bone morphogenetic protein-9 is a circulating vascular quiescence factor. Circ Res. 2008; 102: 914-22

36. Jane J, Dunford B, Kron A, Pittman D, Sasaki T, Li J, et al. Ectopic Osteogenesis Using Adenoviral Bone Morphogenetic Protein (BMP)- 4 and BMP-6 Gene Transfer. Mol Ther. 2002; 6: 464.

37. Reddi AH. Bone morphogenetic proteins: an unconventional approach to isolation of first mammalian morphogens. Cytokine Growth Factor Rev. 1997; 8: $11-20$.

38. Reddi AH. Bone morphogenetic proteins: from basic science to clinical applications. J Bone Joint Surg Am. 2001; 83A: S1-6.

39. Wozney JM. Overview of bone morphogenetic proteins. Spine. 2002; 27: S2-8.

40. Luo J, Sun MH, Kang Q, Peng Y, Jiang W, Luu HH, et al. Gene therapy for bone regeneration. Curr Gene Ther. 2005; 5: 167-79.

41. Wozney JM. The bone morphogenetic protein family: multifunctional cellular regulators in the embryo and adult. Eur J Oral Sci. 1998; 106 Suppl 1: 160-6.

42. Wozney JM, Rosen V. Bone morphogenetic protein and bone morphogenetic protein gene family in bone formation and repair. Clin Orthop. 1998;: 26-37.

43. Huang E, Bi Y, Jiang W, Luo X, Yang K, Gao JL, et al. Conditionally Immortalized Mouse Embryonic Fibroblasts Retain Proliferative Activity without Compromising Multipotent Differentiation Potential. PLoS One. 2012; 7: e32428.

44. Wu N, Zhang H, Deng F, Li R, Zhang W, Chen X, et al. Overexpression of Ad5 precursor terminal protein accelerates recombinant adenovirus packaging and amplification in HEK-293 packaging cells. Gene Ther. 2014; 21: 629-37.

45. Chen X, Cui J, Yan Z, Zhang H, Chen X, Wang N, et al. Sustained high level transgene expression in mammalian cells mediated by the optimized piggyBac transposon system. Genes Dis. 2015; 2: 96-105.

46. Wang N, Zhang W, Cui J, Zhang H, Chen X, Li R, et al. The piggyBac Transposon-Mediated Expression of SV40 T Antigen Efficiently Immortalizes Mouse Embryonic Fibroblasts (MEFs). PLoS One. 2014; 9: e97316.

47. Wen S, Zhang H, Li Y, Wang N, Zhang W, Yang K, et al. Characterization of constitutive promoters for piggyBac transposon-mediated stable transgene expression in mesenchymal stem cells (MSCs). PLoS One. 2014; 9: e94397.

48. Kang Q, Song WX, Luo Q, Tang N, Luo J, Luo X, et al. A comprehensive analysis of the dual roles of BMPs in regulating adipogenic and osteogenic differentiation of mesenchymal progenitor cells. Stem Cells Dev. 2009; 18: 545-59.

49. Luo J, Deng ZL, Luo X, Tang N, Song WX, Chen J, et al. A protocol for rapid generation of recombinant adenoviruses using the AdEasy system. Nat Protoc. 2007; 2: 1236-47.

50. Kong Y, Zhang H, Chen X, Zhang W, Zhao C, Wang N, et al. Destabilization of Heterologous Proteins Mediated by the GSK3beta Phosphorylation Domain of the beta-Catenin Protein. Cell Physiol Biochem. 2013; 32: 1187-99.

51. Gao Y, Huang E, Zhang H, Wang J, Wu N, Chen X, et al. Crosstalk between Wnt/beta-Catenin and Estrogen Receptor Signaling Synergistically Promotes Osteogenic Differentiation of Mesenchymal Progenitor Cells. PLoS One. 2013; 8: e82436.

52. Zhang Y, Chen X, Qiao M, Zhang BQ, Wang N, Zhang Z, et al. Bone morphogenetic protein 2 inhibits the proliferation and growth of human colorectal cancer cells. Oncol Rep. 2014.

53. Chen X, Luther G, Zhang W, Nan G, Wagner ER, Liao Z, et al. The E-F Hand Calcium-Binding Protein S100A4 Regulates the Proliferation, Survival and Differentiation Potential of Human Osteosarcoma Cells. Cell Physiol Biochem. 2013; 32: 1083-96.

54. Zhao C, Wu N, Deng F, Zhang H, Wang N, Zhang W, et al. Adenovirus-mediated gene transfer in mesenchymal stem cells can be significantly enhanced by the cationic polymer polybrene. PLoS One. 2014; 9: e92908. 
55. Lamplot JD, Liu B, Yin L, Zhang W, Wang Z, Luther G, et al. Reversibly Immortalized Mouse Articular Chondrocytes Acquire Long-Term Proliferative Capability while Retaining Chondrogenic Phenotype. Cell Transplant. 2015; 24: 1053-66.

56. Zhang Q, Wang J, Deng F, Yan Z, Xia Y, Wang Z, et al. TqPCR: A Touchdown qPCR Assay with Significantly Improved Detection Sensitivity and Amplification Efficiency of SYBR Green qPCR. PLoS One. 2015; 10: e0132666.

57. Hu N, Jiang D, Huang E, Liu X, Li R, Liang X, et al. BMP9-regulated angiogenic signaling plays an important role in the osteogenic differentiation of mesenchymal progenitor cells. J Cell Sci. 2013; 126: 532-41.

58. Huang E, Zhu G, Jiang W, Yang K, Gao Y, Luo Q, et al. Growth hormone synergizes with BMP9 in osteogenic differentiation by activating the JAK/STAT/IGF1 pathway in murine multilineage cells. J Bone Miner Res. 2012; 27: 1566-75.

59. Si W, Kang Q, Luu HH, Park JK, Luo Q, Song WX, et al. CCN1/Cyr61 Is Regulated by the Canonical Wnt Signal and Plays an Important Role in Wnt3A-Induced Osteoblast Differentiation of Mesenchymal Stem Cells. Mol Cell Biol. 2006; 26: 2955-64.

60. Zhu GH, Huang J, Bi Y, Su Y, Tang Y, He BC, et al. Activation of RXR and RAR signaling promotes myogenic differentiation of myoblastic $\mathrm{C} 2 \mathrm{C} 12$ cells. Differentiation. 2009; 78: 195-204.

61. Liao Z, Nan G, Yan Z, Zeng L, Deng Y, Ye J, et al. The Anthelmintic Drug Niclosamide Inhibits the Proliferative Activity of Human Osteosarcoma Cells by Targeting Multiple Signal Pathways. Curr Cancer Drug Targets. 2015.

62. Wang Y, Hong S, Li M, Zhang J, Bi Y, He Y, et al. Noggin resistance contributes to the potent osteogenic capability of BMP9 in mesenchymal stem cells. J Orthop Res. 2013; 31: 1796-803.

63. Wang J, Zhang $\mathrm{H}$, Zhang $\mathrm{W}$, Huang $\mathrm{E}$, Wang $\mathrm{N}$, Wu $\mathrm{N}$, et al. Bone Morphogenetic Protein-9 (BMP9) Effectively Induces Osteo/Odontoblastic Differentiation of the Reversibly Immortalized Stem Cells of Dental Apical Papilla. Stem Cells Dev. 2014; 23: 1405-16.

64. Li R, Zhang W, Cui J, Shui W, Yin L, Wang Y, et al. Targeting BMP9-Promoted Human Osteosarcoma Growth by Inactivation of Notch Signaling. Curr Cancer Drug Targets. 2014.

65. Liu X, Qin J, Luo Q, Bi Y, Zhu G, Jiang W, et al. Cross-talk between EGF and BMP9 signalling pathways regulates the osteogenic differentiation of mesenchymal stem cells. J Cell Mol Med. 2013; 17: 1160-72.

66. Zhang W, Deng ZL, Chen L, Zuo GW, Luo Q, Shi Q, et al. Retinoic acids potentiate BMP9-induced osteogenic differentiation of mesenchymal progenitor cells. PLoS One. 2010; 5: e11917.

67. Shui W, Yin L, Luo J, Li R, Zhang W, Zhang J, et al. Characterization of chondrocyte scaffold carriers for cell-based gene therapy in articular cartilage repair. J Biomed Mater Res A. 2014; 101: 3542-50.

68. Luo X, Chen J, Song WX, Tang N, Luo J, Deng ZL, et al. Osteogenic BMPs promote tumor growth of human osteosarcomas that harbor differentiation defects. Lab Invest. 2008; 88: 1264-77.

69. Shui W, Zhang W, Yin L, Nan G, Liao Z, Zhang H, et al. Characterization of scaffold carriers for BMP9-transduced osteoblastic progenitor cells in bone regeneration. J Biomed Mater Res A. 2014.

70. Zhang W, Zhang H, Wang N, Zhao C, Zhang H, Deng F, et al. Modulation of beta-Catenin Signaling by the Inhibitors of MAP Kinase, Tyrosine Kinase, and PI3-Kinase Pathways. Int J Med Sci. 2013; 10: 1888-98.

71. Bi Y, He Y, Huang J, Su Y, Zhu GH, Wang Y, et al. Functional characteristics of reversibly immortalized hepatic progenitor cells derived from mouse embryonic liver. Cell Physiol Biochem. 2014; 34: 1318-38.

72. Bi Y, Huang J, He Y, Zhu GH, Su Y, He BC, et al. Wnt antagonist SFRP3 inhibits the differentiation of mouse hepatic progenitor cells. J Cell Biochem. 2009; 108: 295-303.

73. Huang J, Bi Y, Zhu GH, He Y, Su Y, He BC, et al. Retinoic acid signalling induces the differentiation of mouse fetal liver-derived hepatic progenitor cells. Liver Int. 2009; 29: 1569-81.

74. Luo J, Tang M, Huang J, He BC, Gao JL, Chen L, et al. TGFbeta/BMP type I receptors ALK1 and ALK2 are essential for BMP9-induced osteogenic signaling in mesenchymal stem cells. J Biol Chem. 2010; 285: 29588-98.

75. Massague J, Chen YG. Controlling TGF-beta signaling. Genes Dev. 2000; 14 : 627-44.

76. Griffith DL, Keck PC, Sampath TK, Rueger DC, Carlson WD. Three-dimensional structure of recombinant human osteogenic protein 1: structural paradigm for the transforming growth factor beta superfamily. Proc Natl Acad Sci U S A. 1996; 93: 878-83.

77. Cui Y, Jean F, Thomas G, Christian JL. BMP-4 is proteolytically activated by furin and/or PC6 during vertebrate embryonic development. Embo J. 1998; 17: 4735-43.

78. Neugebauer JM, Kwon S, Kim HS, Donley N, Tilak A, Sopory S, et al. The prodomain of BMP4 is necessary and sufficient to generate stable BMP4/7 heterodimers with enhanced bioactivity in vivo. Proc Natl Acad Sci U S A. 2015; 112: E2307-16. 